\title{
Efficacy of single component MTV to measure turbulent wall-flow velocity derivative profiles at high resolution
}

\author{
John R. Elsnab · Jason P. Monty · Christopher M. White · Manoochehr \\ M. Koochesfahani · Joseph C. Klewicki
}

Received: date / Accepted: date

\begin{abstract}
Physical interpretations and especially analytical considerations benefit from the ability to accurately estimate derivatives of experimentally measured statistical profiles. Toward this aim, experiments were conducted to investigate the efficacy of singlecomponent molecular tagging velocimetry (1c-MTV) to measure mean velocity profiles that can be differentiated multiple times. Critical effects here pertain to finite measurement uncertainty in the presence of high spatial resolution. Measurements acquired in fully developed turbulent channel flow over a friction Reynolds number range from 390 to 1800 are used to investigate these issues. Each measured profile contains about 880 equally spaced data points that span from near the edge of the viscous sublayer to the channel centreline. As a result of the high spatial resolution, even very small levels of uncertainty in the data adversely affect the capacity to produce smooth velocity derivative profiles. It is demonstrated that the present 1c-MTV measurements can be differentiated twice, with the resulting profile remaining smooth and accurate. The experimental mean velocity profiles, and their wall-normal derivatives up
\end{abstract}

John R. Elsnab · Jason P. Monty · Joseph C. Klewicki Department of Mechanical Engineering, University of Melbourne,

Parkville, Victoria 3010, Australia

E-mail: john.elsnab@unimelb.edu.au

Christopher M. White · Joseph C. Klewicki

Department of Mechanical Engineering, University of New Hampshire,

Durham, New Hampshire 03824, USA

Manoochehr M. Koochesfahani

Department of Mechanical Engineering, Michigan State University,

East Lansing, Michigan 48824, USA to 2nd order are shown to convincingly agree with existing DNS data, including the apparent variations with Reynolds number.

\section{Introduction}

Advancing aspects of turbulent wall-flow theory, exposing the distinctions between competing theories, or clarifying the effects of flow perturbations are all aided by the ability to experimentally measure wall-normal derivative profiles of statistical quantities. A primary aim here is the reconstruction of the time averaged equations that naturally contain such derivatives. Herein, for specificity, the mean momentum equation is considered. A central difficulty in this regard is that the noise level of the estimated derivative of an experimentally measured profile (with finite uncertainty) is proportionally amplified as the spacing of the points in the profile decreases. Thus, there are rather severe competing effects between the high spatial resolution needed to appropriately characterize the given profile, and the accuracy of its estimated derivatives.

The primary aim of the present study is to investigate whether experiments employing single component molecular tagging velocimetry (1c-MTV) can be utilized to measure first and second derivatives of the mean velocity profile even at very high spatial resolution. Toward this aim, we report on experiments in a canonical turbulent channel flow, and use an analysis involving the time averaged streamwise momentum equation to investigate the degree to which accurate high spatial resolution derivative profile estimates are possible. 


\subsection{Mean Momentum Equation Analysis}

Motivation is gained by noting that, to the authors' knowledge, no previous study has reliably estimated mean second derivatives with respect to the wall-normal coordinate in turbulent wall-flows at high spatial resolution. Indeed, even when manipulating such derivatives from DNS data one often encounters noticeable effects associated with profile uncertainties.

To provide a specific and relevant context for analysis and comparison, we consider the mean dynamics in canonical pressure driven turbulent channel flow. Equation 1 represents the time-averaged streamwise differential force balance for this flow

$\underbrace{-\frac{1}{\rho} \frac{\mathbf{d} p}{\mathbf{d} x}}_{P G}+\underbrace{\nu \frac{\mathbf{d}^{2} U}{\mathbf{d} y^{2}}}_{V F}-\underbrace{\frac{\mathbf{d} \overline{u^{\prime} v^{\prime}}}{\mathbf{d} y}}_{T I}=0$.

Here $\rho$ is the fluid density, $p$ is the pressure, $x$ is the streamwise direction, $\nu$ is the kinematic viscosity, $y$ is the wall-normal direction, $\overline{u^{\prime} v^{\prime}}$ is the Reynolds stress, $P G$ is the pressure gradient, $V F$ is the mean viscous force, and $T I$ is the turbulent inertia (mean inertial force). For this paper, $U$ is the mean streamwise velocity, and lower-case quantities with a "prime" superscript are fluctuations.

Consideration of how the terms in Eq. 1 balance yields the four-layer structure depicted in Fig. 1. This figure effectively conveys the mean differential free body diagram with wall-normal distance (Wei et al, 2005). Here we note that while the Reynolds stress is the time averaged turbulent momentum flux, it is the gradient (divergence) of this flux that establishes the mean distribution of momentum. In layer I, $P G \approx V F$, which is the traditional viscous sublayer ( $T I$ is negligible). In Layer II, $V F \approx T I$, or where the stress gradients balance ( $P G$ is negligible). Layer III is where the $|P G| \approx$ $|V F| \approx|T I|$. Across this layer there is an exchange of leading order terms. This behaviour arises because the gradient of the Reynolds stress passes through zero. The outermost layer, layer IV, is where the leading balance is between the pressure gradient and turbulent inertia ( $V F$ is negligible). When interpreted as a force, the Reynolds stress gradient acts as a net source of momentum on the wallward side of the peak in the Reynolds stress, and a sink of momentum beyond this peak (Klewicki et al, 2007). Regarding the aims of the present investigation, note that without the ability to reliably estimate velocity derivatives the details pertaining to the fundamental properties just described would be very difficult to surmise. Furthermore, sufficiently high quality experimental data can be used to

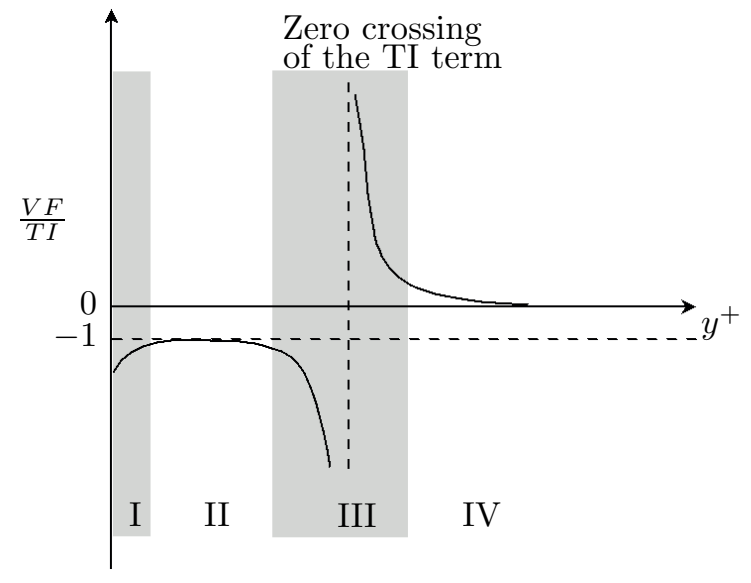

Fig. 1 A sketch of the ratio of terms $V F / T I$ revealing the four layer leading balance structure for turbulent channel flow (adapted from Wei et al (2005)).

advance research that not only pertains to canonical channel flow, but perhaps more significantly, to flows where the mean dynamics have been modified.

In the present experiments, the pressure gradient is determined by measuring the pressure drop in the fully developed region of the test-section. The mean viscous force is determined from the velocity profile, and thus requires high-resolution velocity profile data that are twice differentiable. For this work, the Reynolds stress is calculated by integrating the momentum equation and applying boundary conditions. This yields the familiar exact equation

$-{\overline{u^{\prime} v^{\prime}}}^{+}=1-\frac{y^{+}}{\delta^{+}}-\frac{\mathbf{d} U^{+}}{\mathbf{d} y^{+}}$.

Here the superscript + denotes normalization by the viscous length scale, $\nu / u_{\tau}$, and friction velocity, $u_{\tau}=$ $\sqrt{\tau_{w} / \rho}$, where $\tau_{w}$ is the wall shear stress, $\delta$ is the halfchannel height, $y^{+}=y u_{\tau} / \nu$ is the inner-normalized distance from the wall, and $\delta^{+}=\delta u_{\tau} / \nu$ is the friction Reynolds number. An overbar represents a time average. The efficacy of an adaptation of molecular tagging velocimetry (MTV) is investigated herein relevant to the high resolution quantification of the profile properties associated with equations (1) and (2).

\subsection{Previous MTV Measurements}

MTV is a non-invasive laser based technique that, in many respects, constitutes the molecular counterpart to particle image velocimetry (Koochesfahani et al, 2000). Instead of tracking particles, molecularly tagged regions are tracked. Because particles are replaced by tagged 
molecules dissolved in the fluid, difficulties relating to the particles tracking the flow, density mismatch between the fluid and particles, and particle seeding density are all absent from MTV (Koochesfahani et al, 1997). Furthermore, since MTV does not require particle seeding, its spatial resolution matches that of the imaging system resolution. This makes MTV an attractive technique for experiments requiring high spatial resolution, and accordingly for measuring derivative quantities.

In this regard, Olson et al (2015) obtained wall shear stress and pressure distribution estimates for flow around a circular cylinder at $R e=6000$ by using first and second derivatives of the velocity profile at the surface. These derivatives were estimated by curve-fitting high resolution boundary-layer profile measurements. MTV has also been used to investigate microscale channel flow in the laminar and transitional regimes (Elsnab et al, 2010), laminar flow over an airfoil (Olson et al, 2013), a heated cylinder in cross-flow ( $\mathrm{Hu}$ and Koochesfahani, 2011), and vortex rings (Gendrich et al, 1997). The first MTV study to investigate turbulent wall flows was conducted by Klewicki and Hill (1998). Here they investigated fluctuations in the wall-normal streamwise velocity profiles in a low Reynolds number water flow turbulent boundary layer. Later, Thurlow and Klewicki (2000) investigated turbulent PoiseuilleCouette flow with gas as the working fluid. While showing promise, all of these previous studies were either at relatively low Reynolds number (which generally represents a less stringent measurement condition), or the present desired accuracy of the derivatives (i.e., comparable to that found in DNS) was not required. The objective of this work is to investigate if 1c-MTV can obtain high spatial resolution mean velocity profiles that are twice differentiable in a manner similar to current DNS profile data.

\section{Methods and Procedures}

\subsection{Facility}

A rectangular water channel with test-section dimensions $60 \mathrm{~mm} \times 750 \mathrm{~mm} \times 9000 \mathrm{~mm}(\mathrm{H} \times \mathrm{W} \times \mathrm{L})$ was used for the experiments. Here $H$ is the channel height, $W$ is the channel width and $L$ is the length. For turbulent flows, Dean (1978) analyzed a large set of available data and found that three-dimensional effects slightly disturb the flow near the centerline if the aspect ratio, $W / H$, is less than 7. An aspect ratio of 12.5 is believed to accurately replicate two-dimensional channel flow, as a similar aspect ratio channel was successfully employed in the studies of Monty (2005). Furthermore, Monty and
Chong (2009) showed that experimental data obtained with a channel aspect ratio of 11.7 exhibit excellent agreement with DNS data at similar $\delta^{+}$.

A schematic of the experimental facility is presented in Fig. 2. The test-section is made of precision machined cast aluminium plates (flat within $0.127 \mu \mathrm{m}$ with a maximum surface roughness of $0.5 \mu \mathrm{m}$ ), and stainless steel sidewalls that are precision CNC machined to set the channel height. Nine, one meter long sections are joined to form the test-section. Precision clamps join the individual sections such that all interior surfaces are flush. Before the flow enters the test-section, it passes through three screens, two with an open area of $72 \%$, and then one fine screen with an open area of $58 \%$. All of the screens have a wire diameter of $0.8 \mathrm{~mm}$, and the spacing between screens is $550 \mathrm{~mm}$. Following the screens, the flow passes through a contraction with an area reduction factor of 6.66. Fused silica optical windows installed flush into the channel floor and ceiling allow for laser beam access. Similarly, windows in the vertical side walls allow for camera viewing. These windows are located at $130 H$ downstream of the contraction, as noted in Fig. 3. The fluid flow is driven by a centrifugal pump that is controlled with a precision variable frequency drive.

According to Monty (2005), one can expect fully developed turbulent flow based upon mean velocity for development lengths exceeding $130 H$. Thus, the entrance length for this study ensures that the flow reaches a fully developed state in the streamwise direction for all $\delta^{+}$investigated. Wall pressure taps, $1 \mathrm{~mm}$ in diameter, located at $x / H=31.7,48.3$, and 65 are used to measure the pressure drop. The downstream pressure port located at $x / H=131.7$ is used as the reference pressure for the three $\Delta p$ sensors. The pressure ports are located in the linear pressure drop region for all $\delta^{+}$, thus eliminating the need to account for the entrance pressure loss. The downstream tap is located at $x / H=20$ from the channel exit so that expansion effects are not present. A linear curve-fit using the three $\Delta p$ measurements as a function of streamwise distance is performed to calculate the pressure gradient. The fluid temperature is measured using shielded T-type thermocouples at the inlet and outlet of the test-section. Dynamic viscosity and fluid density as a function of temperature are obtained using correlations provided by Nagashima (1977) and Hyland and Wexler (1980), respectively. The correlations are evaluated at the average temperature. The friction velocity is obtained using the fluid density and pressure gradient, as the wall-shear stress is related to the pressure gradient through an integral momentum balance in the streamwise direction. The uncertainty in 


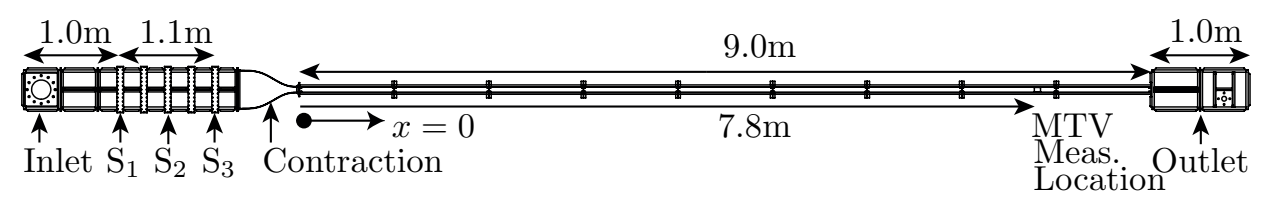

Fig. 2 Schematic of the experimental facility. $S_{i}$ denote the screen locations.

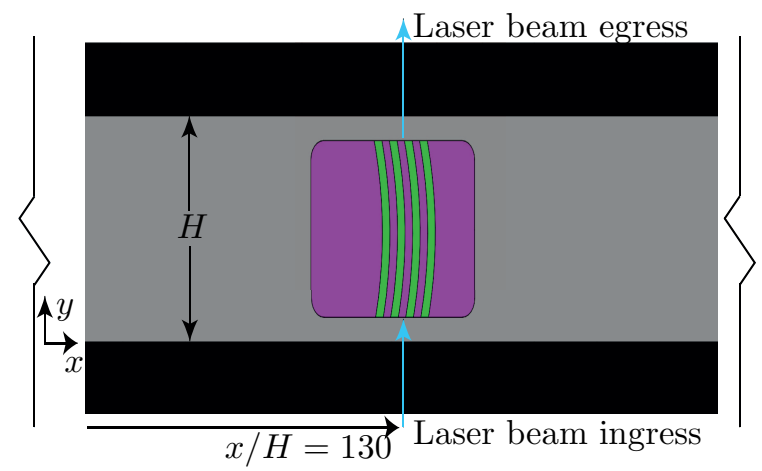

Fig. 3 Schematic of the MTV portion of the facility. Fluid flow is from left to right and the green lines represent a view of the deformed image of the molecularly tagged lines.

$u_{\tau}$ is approximately $1-2 \%$ as assessed using the method described by Moffat (1988).

\subsection{Molecular Tagging Velocimetry}

An ultraviolet (UV) source is used to excite the tracer molecules that are dissolved in the water. The line pattern is subsequently imaged at an initial time, $t_{o}$, and then again at a delayed time, $t_{o}+\Delta t$, with both images being captured within the emission lifetime of the tracer. Here $\Delta t$ is the time delay between the two successive images. In processing, the two images are compared and the velocity field is determined from the measured Lagrangian displacement of the tagged fluid molecules. The displacement can be determined any number of ways. This includes using direct cross-correlation methods (Gendrich and Koochesfahani, 1996), spline fitting (Sadr and Klewicki, 2003), and template matching (Ramsey and Pitz, 2011). An account of the history of the MTV technique and its various implementations is given by Koochesfahani and Nocera (2007).

While the high spatial resolution afforded by $1 c-$ MTV facilitates the accurate estimation of derivatives, the very close data spacing renders derivative quantities especially sensitive to measurement uncertainty. Recognizing that each imaging system has its individual attributes, a limitation of the intensified CCD camera used here is the presence of a fixed background spatial pattern, referred to as the chicken wire pattern. This pattern introduces a low level spatial "noise" into the acquired images, subsequently making it difficult to obtain derivatives with high accuracy. The pattern is referred to as chicken wire because it is hexagonal in shape, which is due to the hexagonal geometry of the coupling fibers between the CCD and intensifier arrays. This fixed pattern is due to fiberoptic coupling, and thus is not present when using optical coupling. On the other hand, fiberoptic coupling tends to be more efficient.

An intensified CCD camera was used in the present experiments for two reasons. The first is that the intensifier produced the requisite signal to noise needed to accurately determine the MTV line positions, and the second is that this kind of camera allows one to acquire image pairs on the same imaging array for each instantaneous measurement. This capability to capture the undeformed and deformed MTV line images for each instantaneous measurement (as opposed to using the average undeformed image) is important for experiments that are unsteady, or when other modifications, such as the addition of particles into the flow, influence the undeformed line images from one instant to the next (Sadr and Klewicki, 2003). Aspects of the procedure described by Bohl (2002) to deal with ICCD chicken-wire artifacts are used to remove a majority of the fixed spatial pattern. Similar procedures were also used in the vorticity measurements of Bohl and Koochesfahani (2009). To implement this procedure, one acquires two correction images: (1) a darkfield image and (2) a flatfield image. The goal of the flatfield normalization is to remove artifacts caused by variations in the pixel-to-pixel nensitivity of the detector, i.e., remove the systematic error largely associated with the chicken wire pattern. The darkfield image is the amount of signal output by the detector when there is no incident light. The flatfield image is obtained using an opaque glass plate uniformly illuminated with LED back-lighting. This procedure does not correct for variations in the laser intensity along the line. For the data density of the present measurements, accurately estimating second derivatives required that the net effect of these background aberrations had to be reduced to about a $\pm 1 \mu \mathrm{m}$ uncertainty in the mean displacements of the molecularly tagged lines.

The photo-sensitive MTV tracer molecules in the fluid are tagged using a fourth harmonic Pro-350 Nd:YAG 


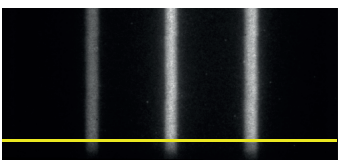

$t=t_{o}$

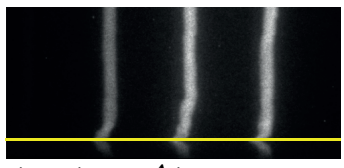

$t=t_{o}+\Delta t$
Fig. 4 Instantaneous image of undeformed and deformed MTV lines. The fluid flow is from left to right. The solid yellow line represents the wall location and only $1 / 5$ of the image height is presented.

laser $(266 \mathrm{~nm})$ manufactured by Spectra Physics. Individual laser lines are formed using an array of pinholes that are $0.75 \mathrm{~mm}$ in diameter with a pitch ranging from $2-4 \mathrm{~mm}$. The beam blocker is precision $\mathrm{CNC}$ machined out of aluminium. The concentration of the individual constituents making up the MTV solution $(1-\mathrm{BrNp} \cdot \mathrm{M} \beta-\mathrm{CD} \cdot \mathrm{ROH})$ is $2 \times^{-4} \mathrm{M}$ for the cyclodextrin, $\mathrm{M} \beta-\mathrm{CD}, \sim 10^{-5} \mathrm{M}$ for the lumophore, 1-BrNP, and 0.05 $\mathrm{M}$ for the alcohol concentration, $\mathrm{ROH}$ (Koochesfahani and Nocera, 2007). These values are recommended by Gendrich et al (1997) to obtain $90 \%$ of the maximum emitted intensity when using cyclohexanol as the alcohol. Due to the low concentrations, any variations in density or viscosity of the carrier fluid (water) were not detectable. MTV images are captured using a $1024 \times$ 1024 pixel Princeton Instruments PI:MAX4 ICCD camera. Each pixel is $12.8 \times 12.8 \mu \mathrm{m}$. Typically, $2000-2500$ image pairs were acquired at a rate of $5 \mathrm{~Hz}$ with $3-6$ laser lines per image. Since the flow is fully developed, the mean profile at each line position is the same, and thus, the over all sample size for a given experiment is the product of the image pairs times the number of lines. Two point correlation in the streamwise direction indicate that the profiles at each $x$ location have non-zero correlation, and thus are not entirely independent. The time delay between successive images in an image pair ranged between $556-2619 \mu$ s with an exposure time for the undeformed and deformed images of $50 \mu \mathrm{s}$ and $75 \mu \mathrm{s}$, respectively. In viscous units, the undeformed image exposure time is $[8.5,21.0,47.2,162] \times$ $10^{-3}$ and for the deformed image, $[12.7,30.2,70.8,243] \times$ $10^{-3}$, respectively for the progression from low to high $\delta^{+}$. The undeformed image is acquired $5 \mu \mathrm{s}$ after laser emission. Examples of instantaneous undeformed and deformed line images in the lower $1 / 5$ of the channel flow are presented in Fig. 4.

\subsection{Data Reduction}

Post-acquisition image pre-processing utilizes a spatial smoothing step that employs a median filter with five streamwise data points at each wall-normal location
(Caso and Bohl, 2014). To remove the aforementioned chicken wire background pattern on the ICCD, each pixel in the undeformed and deformed images are corrected according to

$$
I_{C}\left(x_{i}, y_{j}\right)=\frac{I\left(x_{i}, y_{j}\right)-D F\left(x_{i}, y_{j}\right)}{F F\left(x_{i}, y_{j}\right)-D F\left(x_{i}, y_{j}\right)}
$$

Here $I_{C}$ is the normalized image, $I$ is the instantaneous image, $D F$ is the darkfield image, and $F F$ is the flatfield image. The flatfield image is captured at the same intensifier gain as used to capture the images of the undeformed and deformed lines.

The bulk displacement is determined by an FFTbased cross-correlation at each wall-normal pixel location. Sub-pixel displacement is determined by curve fitting a second-order polynomial through the peak in the correlation distribution. Essentially identical results were also obtained using a third-order polynomial. The instantaneous deformed images are cross-correlated with their respective undeformed image. The $95 \%$ confidence interval for measurements using the present camera is 0.3 pixel. This estimate is based upon the method outlined by Bohl (2002). The measurement error for the camera is determined by imaging a static target that consists of white vertical lines separated by black lines. The line width of the white lines is set to approximately that of the MTV images, around 18 pixel. Crosscorrelating the static image allows for the measurement error to be determined. Ideally, this analysis should yield zero displacement. By cross-correlating over 100 images, the distribution of errors about the perfect (zero displacement) condition is obtained. The error is uniformly distributed, and thus the rms measurement uncertainty is estimated to be half the $95 \%$ level or 0.15 pixel (Caso and Bohl, 2014). The estimated sub-pixel accuracy of the mean displacement is $0.15 / \sqrt{N}$ pixel, where $N$ is the number of profiles used in the average. The corresponding uncertainty in the mean centerline velocity was then determined to be $0.25 \%$. The uncertainty of the inner normalized mean velocity at the centerline is $1 \%$, and about $1.5 \%$ of a viscous unit for the spatial position. The absence of wall glare from the laser emission in the acquired images allows the use of the tagged line reflection to determine the position of the wall. Namely, the apparent position of reflection in the displacement profile locates the wall. 


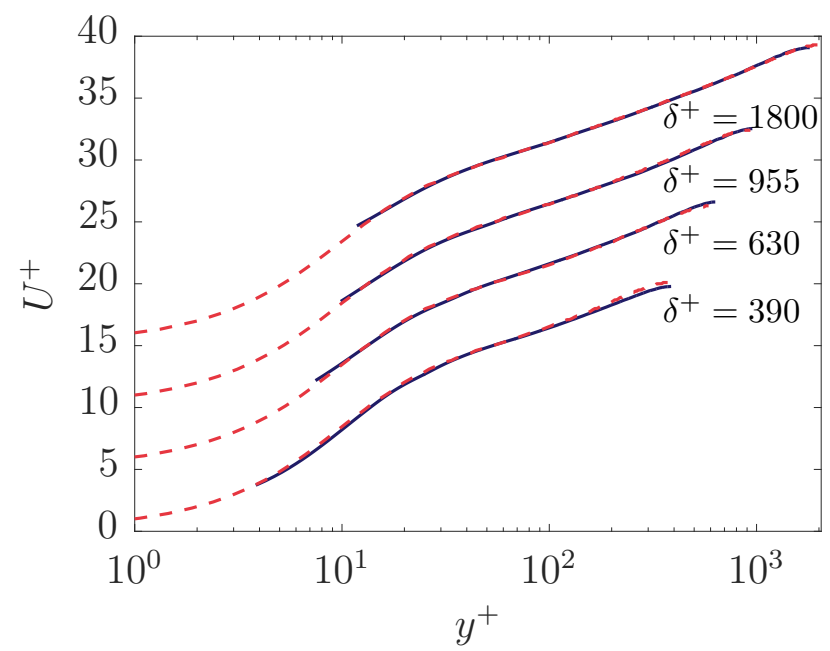

Fig. 5 Inner normalized mean velocity profiles along with a comparison between DNS profiles at similar Reynolds numbers. Note that the profiles are vertically shifted by 5 between each $\delta^{+}$. Description: solid line, experimental profiles and dashed line, DNS profiles. Note that the 1-cMTV method produces a velocity measurement at every pixel.

\section{Results}

\subsection{Mean and RMS Streamwise Velocity}

Inner normalized mean velocity profiles are presented and compared against DNS data in Fig. 5. The DNS data are from Moser et al (1999) at $\delta^{+}=392$ and 590, and Hoyas and Jimenez (2006) at $\delta^{+}=934$ and 2004. At $\delta^{+}=390$, the experimental results show very good agreement with the DNS, except for a slight variation in the wake region. For the other $\delta^{+}$profiles, the experimental results are in excellent agreement with the DNS over the entire profile. Here we note that each of the experimental profiles contains about 880 data points, which considerably exceeds the number of points in any of the DNS profiles of Fig. 5. For each MTV profile the data are equally spaced, and cover a domain that starts about $0.3 \mathrm{~mm}$ from the wall and extends to the channel centerline at $y=30 \mathrm{~mm}$. This yields, for example, an inner-normalized spacing of about $\Delta y^{+}=2.1$ for the data at the highest Reynolds number. The first measurement position near the wall is determined by the quality of the data in that region. This position is nominally fixed in physical units, and thus, as indicated in the figures of this section this position moves to increasing $y^{+}$locations with increasing $\delta^{+}$.

Before discussing the streamwise velocity rms profiles for varying friction Reynolds numbers, the result for the highest Reynolds number case, $\delta^{+}=1800$, is highlighted in Fig. 6 to illustrate the effect of spatial attenuation. This effect is evident since the measured rms data have lower amplitude than the DNS data. This attenuation is caused by a spatial averaging effect that can be corrected using the technique described by Lee et al (2016) and is attributed to the depth of focus (DOF) of the lens. Here the MTV technique is acting similar to a hot-wire sensor in terms of attenuation caused by finite spatial resolution in the spanwise direction. This attenuation effect is distinct from the volumetric attenuation typically experienced by PIV or LDV measurements. Using the estimated MTV line thickness in $z$ (full-width at half of the maximum intensity) as the characteristic length $(0.65 \mathrm{~mm})$ associated with the "sensor" attenuation, the spatially corrected MTV results nearly replicate those of the DNS. The corrected profile is the sum of the attenuation obtained from Lee et al (2016), $S A_{z}$, and $U D$ based rms profiles. Here $U D$ refers to profiles that are generated from a separate deformed and undeformed image pair.

The length scale associated with the attenuation can be argued to be appropriate if it does not change with Reynolds number. This condition was determined to hold in the present data over the range $600<\delta^{+}<$ 1800. At $\delta^{+}=390$, the spatial correction technique described by Lee et al (2016) loses relevance since the spanwise spatial resolution of the DNS nearly matches that of the experiment. In a similar vein, the hot-wire correction proposed by Smits et al (2011) also does not apply for $z_{s r}^{+}<10$ (over correction), although no lower bound was listed in their paper, where $z_{s r}^{+}$is the spatial resolution in the spanwise direction. Based upon DNS studies, if the spatial resolution in the spanwise direction is $z_{s r}^{+}<4$, the attenuation becomes negligible (Moser et al, 1999; Hoyas and Jimenez, 2006). This same behavior is also seen in hotwire studies of spatial attenuation (Johansson and Alfredsson, 1982; Klewicki and Falco, 1990). For the present $\delta^{+}=390$ flow the spanwise spatial resolution is $z_{s r}^{+}=5$, and thus, as expected, these measurements experience essentially negligible attenuation. The corrected streamwise rms velocity profiles are presented in Fig. 7. For the lower $\delta^{+}$case, the MTV data are nearly identical to the DNS data, while the results for the other $\delta^{+}$are in very good agreement with the DNS profiles.

The 1c-MTV technique can be susceptible to a bias error in the presence of shear due to the velocity component parallel to the line of tagged molecules. This error approaches zero as the wall is approached (Hill and Klewicki, 1996; Koochesfahani and Nocera, 2007). The error is given by $\Delta u=v \partial u / \partial y \Delta t$ (Hill and Klewicki, 1996), therefore, the error is reduced by decreasing $\Delta t$. However, $\Delta t$ cannot be made arbitrarily small since it needs to be large enough for the resulting displace- 


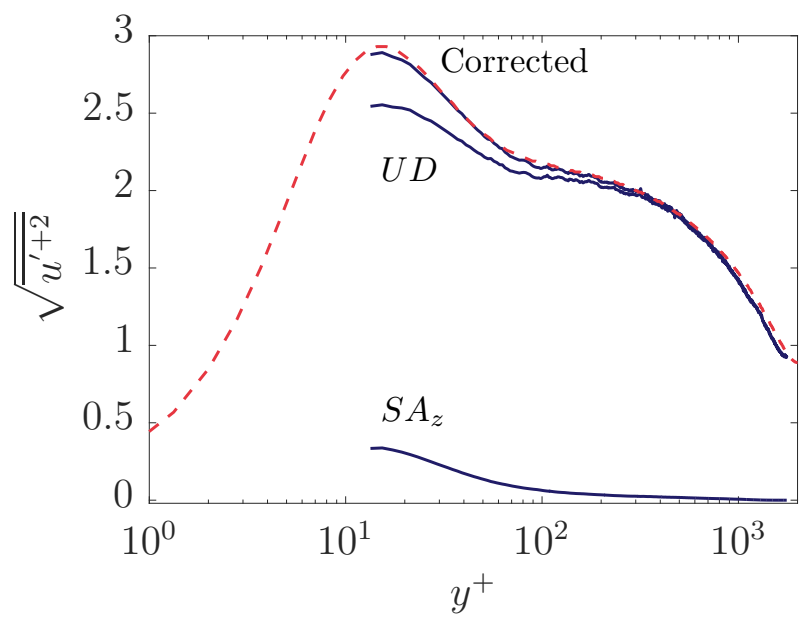

Fig. 6 Experimental streamwise velocity rms profile for $\delta^{+}=1800$ that illustrates the effect of signal attenuation caused by spatial averaging. $S A_{z}$ is the spatial attenuation correction of Lee et al (2016), and $U D$ is the rms profile found from the correlation between the instantaneously captured deformed and undeformed line images. The corrected profile is given by $U D+S A_{z}$, and the dashed line is the DNS profile at $\delta^{+}=2004$. Note that for the given finite width line the $S A_{z}$ profile decreases in magnitude with decreasing $\delta^{+}$, and is negligible at $\delta^{+}=390$.

ment to be measured with adequate accuracy. This error will not affect the average streamwise velocity measurements, since the mean wall-normal velocity is zero in channel flow. It does, however, introduce error into Fig. 7. Consistent with previous 1c-MTV measurements in shear flows, however, the present comparisons with DNS data suggest that this error is small.

MTV data can also experience a temporal filtering effect associated with the averaging that occurs over the exposure period of the ICCD. This effect is physically similar to that found by Pouya et al (2015) in the context of experimentally estimating the intensity of Brownian fluctuations with finite sampling time sensors. In this regard, there are trade-offs associated with the camera time delay and exposure duration relative to accurately measuring the $u^{\prime}$ profiles. These pertain to the camera exposure being sufficiently long to ensure an acceptable signal to noise ratio, but during this time there is turbulent activity. In the present study, the focus was on mean profile measurements, and no effort was made to optimize the MTV image capture attributes relative to measuring the streamwise velocity fluctuations. This, and the other factors noted above, gain importance when considering how to optimize measurements of $u^{\prime}$, or instantaneous estimates of $\partial u / \partial y$.

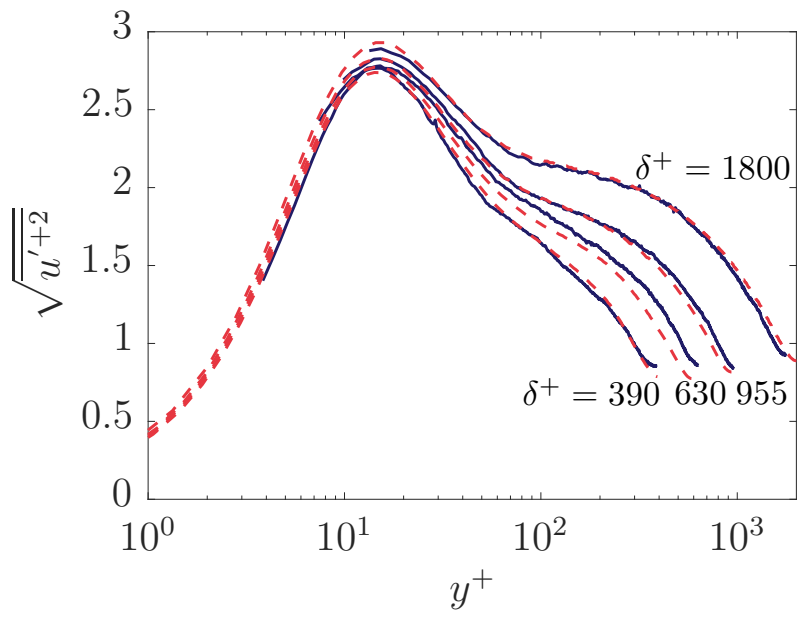

Fig. 7 Streamwise velocity rms profiles along with a comparison between DNS profiles. Description: solid line, experimental profiles and dashed line, DNS profiles.

\subsection{Velocity Gradient Comparisons}

The measured mean velocity gradient $\left(\mathbf{d} U^{+} / \mathbf{d} y^{+}\right)$data exhibit very good agreement with the DNS data for $y^{+} \gtrsim 20$ as shown in Fig. 8. The derivatives are obtained using a third-order polynomial Savitzky-Golay filter (Savitzky and Golay, 1964) with the number of points ranging from $11-31$. Here we note that recalculating $\mathbf{d} U^{+} / \mathbf{d} y^{+}$from the present data using either first or second order forward finite difference schemes yields results that are nearly identical to those in Fig. 8. This agreement between the finite difference and the Savitzky-Golay filter methods would, however, not be observed if the underlying mean profile data had greater uncertainty. Namely, the finite difference result would exhibit significant point-to-point scatter. There are some deviations between the DNS and measured mean velocity gradient profiles, and these cause noticeable effects in the second derivative profiles. One such anomaly is evident in the $\delta^{+}=390$ profile for $20<y^{+}<30$. Despite this, the profile retains agreement with the DNS profile. There is also an anomaly in the $\delta^{+}=955$ profile near $25<y^{+}<45$. The trends for the rest of the $\delta^{+}$ profiles are consistent with the DNS data for $y^{+}>20$.

The observed near-wall error is attributed to the inability of the MTV data to accurately capture the curvature of the near-wall mean profile, and this becomes apparent by examining the first and second derivative profiles. By any typical experimental measurement standard, however, the mean velocity data themselves agree quite well with the DNS data from $10<y^{+}<20$. Another source of difference between the DNS and present experimental results is that the Reynolds numbers of 
comparable profiles do not exactly match. The differences in Reynolds number are apparent, say in Fig. 10, by the two profiles basically tracking parallel to each other, but exhibiting a consistent $y^{+}$shift. Other types of profile deviations are generally attributable to the measurement uncertainties.

A comparison between high quality PIV channel flow data from Kwon et al (2014) with the MTV and DNS data at $\delta^{+} \simeq 1000,2000$ is shown in Fig. 8. It is clear that the MTV data follows the DNS profile much closer to the wall $\left(y^{+}>20\right)$, whereas the PIV data are Reynolds number dependent. For the PIV data at $\delta^{+}=1000$, the agreement with DNS data starts at about $y^{+}=100$, but for $\delta^{+}=2000$, the PIV and DNS data begin to agree near $y^{+}=160$. The spacing between each data point for the PIV data is 7.6 and 15.3 viscous units at the two $\delta^{+}$. This spacing is a factor of 7 greater than the MTV data at similar $\delta^{+}$. It is critical for the MTV data to accurately represent the mean derivative as close to the wall as possible since the Reynolds stress is calculated using the integrated momentum equation. Furthermore, in order to fully utilize the mean momentum balance to investigate the flow structure, data has to be obtained interior to the peak in Reynolds stress.

The mean viscous force (second derivative) is shown in Fig. 9, where the derivatives are obtained using the same number of points and methods as the first derivative. The small anomalies in the mean velocity gradient are amplified in the second derivative, e.g., see the $\delta^{+}=390$ profile around $20<y^{+}<30$, but the profiles are still in good agreement with the DNS data for $y^{+}>20$. The DNS second derivative profiles shown in Fig. 9 are calculated from the mean velocity gradient published in the respective datasets. These second derivative profiles also exhibit some noticeable effects caused by the finite accuracy of the DNS, and this effect becomes more apparent if the mean velocity profile is differentiated twice.

Reynolds shear stress profiles were obtained from the once integrated momentum equation (Eq. 2), and are presented in Fig. 10. As can be seen, these are in very good agreement with the DNS for $y^{+} \gtrsim 10$, both in trend and magnitude. Over the given $\delta^{+}$range, the location of the peak value, $y_{m}^{+}$, moves outward at $y_{m}^{+} \sim \delta^{+0.343}$, and for the DNS data at $\delta^{+0.31}$. This slight difference between the measured and DNS based exponent stems from noise in the Reynolds stress profile. This noise makes it difficult to precisely determine the maximum, and especially as $\delta^{+}$increases. Note that the exponent is lower than the $\delta^{+0.5}$ value presented by Sreenivasan (1989) at high $\delta^{+}$, as well as the transitional $\delta^{+}$results of Elsnab et al (2011). The deviation of

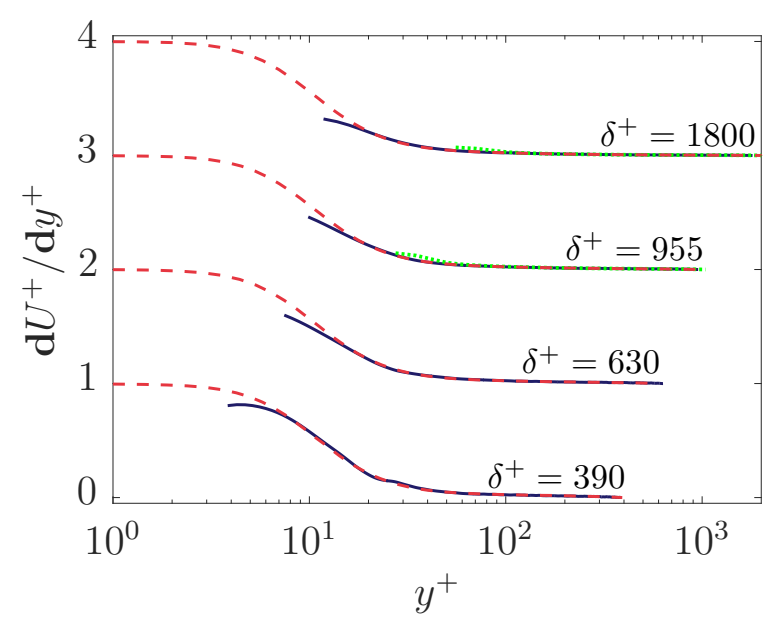

Fig. 8 Mean velocity gradient profiles, $\mathbf{d} U^{+} / \mathbf{d} y^{+}$, along with a comparison with similar Reynolds number DNS profiles. The profiles are shifted vertically by 1.0 between each $\delta^{+}$ profile. Solid lines give the experimental MTV profiles, dotted lines give the PIV profiles, and dashed lines give the DNS profiles. Note that near the wall the discrepancy between the measured and DNS profiles is caused by the high sensitivity to the mean profile shape in this region of the flow.

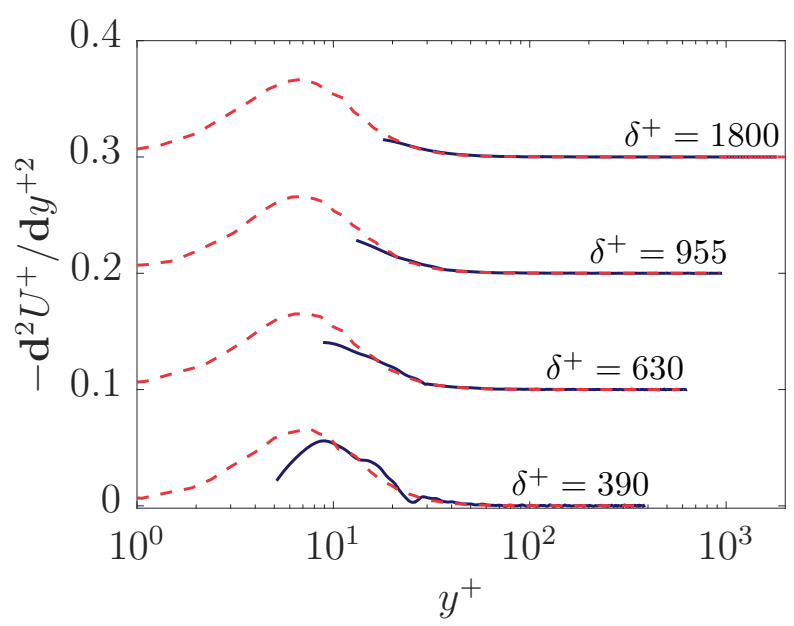

Fig. 9 Measured mean viscous force profiles as compared with similar Reynolds number DNS profiles. Note that the different $\delta^{+}$profiles are shifted vertically by 0.1 . Solid lines denote the experimental profiles and dashed lines denote that DNS profiles.

the measured and DNS profiles in Fig. 10 near the wall is due to the similar deviations in the $\mathbf{d} U^{+} / \mathbf{d} y^{+}$profiles, since the $\overline{u^{\prime} v^{\prime}}$ profiles are determined from Eq. 2.

The experimental version of Fig. 1 is presented in Fig. 11. The data in this figure essentially involves taking the ratio of two quantities that depend on the second $y$ derivative of the mean velocity profile. In terms of accurately reflecting the properties of the mean momentum balance, the measured ratios are in very good 


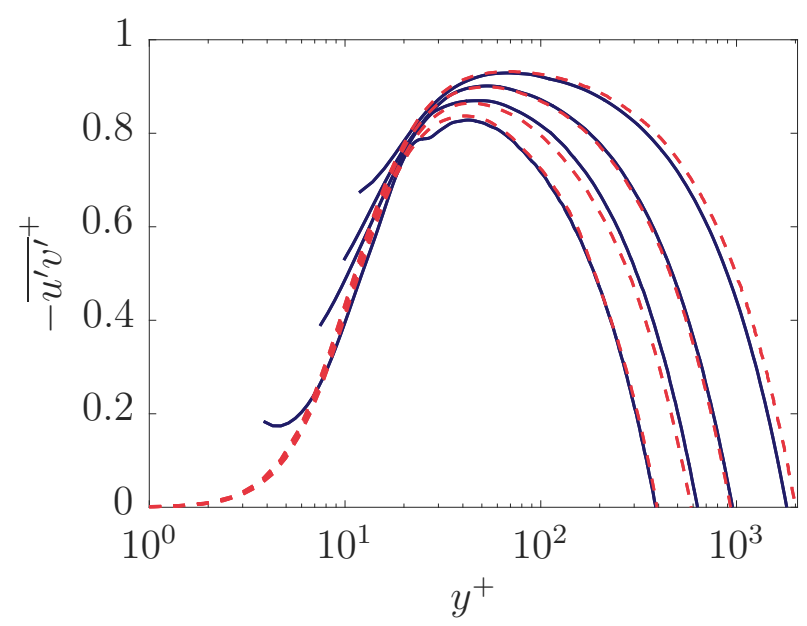

Fig. 10 Reynolds stress profiles obtained from the onceintegrated momentum equation, along with a comparison between DNS profiles. Solid lines denote the experimental profiles, and dashed lines denote the DNS profiles. The $y^{+}$end point in each profile gives the respective Reynolds number, $\delta^{+}$.

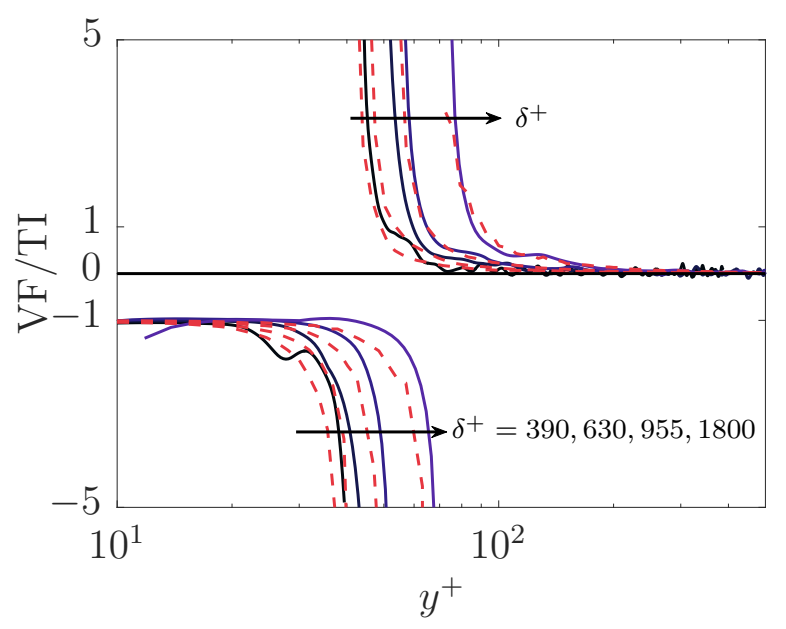

Fig. 11 Ratio of the viscous force to turbulent inertia for the present experimental profiles. Solid lines denote the experimental profiles and dashed lines denote the DNS profiles.

agreement with the DNS profiles. The data of Fig. 11 provide strong evidence that twice differentiable data are well-approximated using the present 1c-MTV technique - even in the outer region of the flow where both the numerator and denominator of the ratio in Fig. 11 become very small.

\section{Conclusion}

Experiments in turbulent channel flow were conducted to develop the ability to measure mean profile derivatives using high spatial resolution 1c-MTV. High reso- lution velocity profiles were obtained over the channel half-height of $30 \mathrm{~mm}$ at four separate Reynolds numbers. Each of the profiles contained about 880 equally spaced velocity measurements, and thus under the lowest spatial resolution condition $\left(\delta^{+}=1800\right)$ the spacing between points was only about 2.1 viscous units. By employing the procedures described herein, the mean velocity, the derivatives of this profile (up to second order), and the associated Reynolds stress profiles were shown to exhibit very good agreement with available DNS data for $y^{+}>20$. To the authors' knowledge, the present experiments document the first instance of precisely estimating second derivative profiles at high resolution in a turbulent wall-flow using experimentally measured mean profiles.

\section{Acknowledgement}

This study was supported by Australian Research Council grant numbers DP120101467 and LE130100088. The authors also thank Dr. Hoyas, Dr. Jimenez, Dr. Kim, Dr. Mansour, and Dr. Moser for making their DNS data available.

\section{Appendix}

Required Resolution and Displacement Accuracy

Overall, the accuracy of the profiles of the terms in the mean momentum equation (or any other mean equation that has derivatives) depends upon the flow situation. In the present study, the mean equation for the canonical flow is examined. In a flow that is perturbed from the canonical state, the nature of the perturbations will generate features in the instantaneous dynamics that impart their signature on the mean profile. Reasonably then, one needs the capacity to resolve these features. Thus, as a general operational rule, measurements must capture derivatives over only a few viscous lengths. This is why we sought to generate profiles containing over 850 data points each, as this will provide the desired resolution, essentially independent of perturbation, over the given Reynolds number range of the facility.

More generally, to quantify the spatial resolution required to obtain accurate derivatives, one can differentiate the data using various step sizes $\left(\Delta y^{+}\right)$. For $\delta^{+}=955$, the step size varied from $1-4$ viscous units in the wall-normal direction and at $\delta^{+}=1800, \Delta y^{+}$was varied between $2-8$. The data shown in Fig. 12 for both $\delta^{+}=955,1800$ indicate that if $\Delta y^{+}>2$, the accuracy of the derivatives diminishes in the near-wall region. However, for $y^{+}>100$, the deviation is not pronounced 
and increasing the step size can smooth the data. In the outer layer, the increase in step size is acting as a filter. As $y^{+} \rightarrow \delta^{+}$, the gradient approaches zero; therefore, any error in the velocity introduces noise that propagates when determining the Reynolds stress and subsequent gradients.

Not surprisingly, the requisite spatial resolution is a function of wall-normal position. This is made apparent by the resolutions employed in DNS. At $y^{+}=10, \delta^{+}=$ 934, the spacing is about 1 viscous unit, but increases to $\Delta y^{+}=3.3$ at $y^{+}=100$. At $\delta^{+}=2004$, the spacing is 1.5 viscous units at $y^{+}=10$ and 4 at $y^{+}=100$. The experimental data are at these resolutions in the near-wall region ( 1 and 2 viscous units for $\delta^{+}=955$ and 1800) and are constant across the channel half-height. From Fig. 12, when $\Delta y^{+}$exceeds that of the DNS, the first derivative loses accuracy. Therefore, the spatial resolution significantly impacts the first derivative, especially for $y^{+}<100$. Based upon the experimental results and the wall-normal spacing of the DNS, the minimum required spatial resolution at a given $y^{+}$can be estimated from the DNS data. The minimum spatial resolution required for the mean momentum balance is based upon the first derivative since the Reynolds stress is obtained from the integrated momentum equation. The second derivative essential follows the first derivative in terms of spatial resolution requirements as shown in Fig. 13.

To set a baseline on the required velocity accuracy, the $U^{+}$profile from the $\delta^{+}=934$ DNS was differentiated using a forward finite difference scheme with the result being compared to the published $\mathbf{d} U^{+} / \mathbf{d} y^{+}$ derivative presented in the DNS database. The resulting profiles are presented in Fig. 14. It is clear that any noise in the velocity data results in a noisy derivative since it is assumed that the spatial resolution of the DNS simulation is sufficient. The standard deviation about the published derivative and the forwarddifference calculated at $\delta^{+}=934$ is 0.014 . To determine the level of accuracy required for the experimental mean velocity data, white noise is added at various signal-to noise ratios. The data presented in Fig. 14 are for signal to noise ratios (SNR) from $60-40$. The SNR for the original profiles is approximately 90 . The error increases with decreasing SNR as shown in Fig. 14, and for the aforementioned SNRs, the standard deviations are $[0.021,0.065,0.205]$, respectively.

Based upon the data presented in Fig. 14, it is not possible to provide a maximum uncertainty in the mean velocity that allows for accurate determination of derivatives. Smoothing derivative schemes can, however, be used to mitigate the effect of noisy data, e.g., noise reducing Richard extrapolations schemes. The effect of noise in the displacement profile increases when cal-

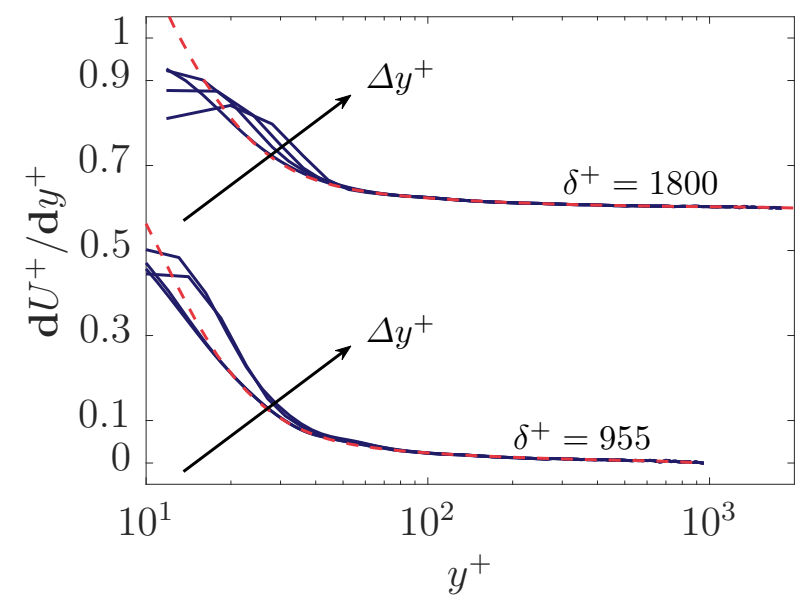

Fig. 12 Mean velocity gradient profiles as function of $\Delta y^{+}$ for $\delta^{+}=955,1800$. Note that the profiles are shifted vertically by 0.6 between each $\delta^{+}$. Solid lines give the experimental profiles and dashed lines give the DNS profiles.

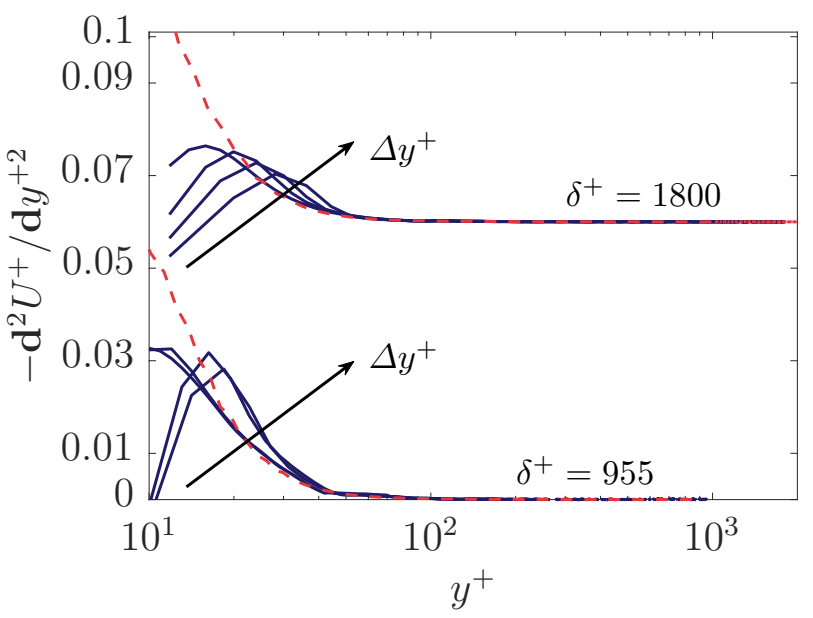

Fig. 13 Mean viscous force profiles as function of $\Delta y^{+}$for $\delta^{+}=955,1800$. Note that the profiles are shifted vertically by 0.06 between each $\delta^{+}$. Solid lines give the experimental profiles and dashed lines give the DNS profiles.

culating the second derivative. Overall we summarize that accurate velocity data are required across the entire half-channel, but spatial resolution can be relaxed for $y^{+} \gtrsim 100$.

\section{References}

Bohl D (2002) Experimental study of the 2-D and 3-D structure of a concentrated line vortex. PhD thesis, Michigan State University, East Lansing, Michigan

Bohl D, Koochesfahani M (2009) MTV measurements of the vortical field in the wake of an airfoil pitching 


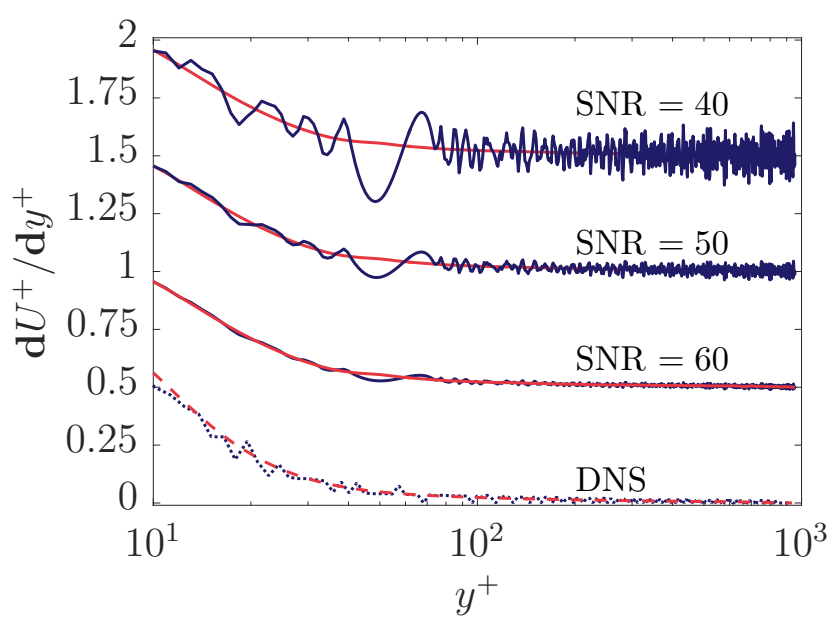

Fig. 14 Mean velocity gradient profiles as function of SNR for $\delta^{+}=955$. Note that the profiles are shifted vertically by 0.5 for each SNR. Solid lines give the experimental profiles and dashed/dotted lines give the DNS profiles at $\delta^{+}=934$.

at high reduced frequency. J Fluid Mech 620:63-88

Caso M, Bohl D (2014) Error reduction in molecular tagging velocimetry via image preprocessing. Exp Fluids 55:1802-1811

Dean RB (1978) Reynolds number dependence of skin friction and other bulk flow variables in twodimensional rectangular duct flow. J Fluid Eng 100:215-223

Elsnab J, Maynes D, Klewicki J, Ameel T (2010) Mean flow structure in high aspect ratio microchannel flows. Exp Thermal Fluid Sci 34:1077-1988

Elsnab J, Klewicki J, Maynes D, Ameel T (2011) Mean dynamics of transitional channel flow. J Fluid Mech 678:451-481

Gendrich CP, Koochesfahani MM (1996) A spatial correlation technique for estimating velocity fields using molecular tagging velocimetry (MTV). Exp Fluids $22: 67-77$

Gendrich CP, Koochesfahani MM, Nocera DG (1997) Molecular tagging velocimetry and other novel applications of a new phosphorescent suparmolecule. Exp Fluids 23:361-372

Hill RB, Klewicki JC (1996) Data reduction methods for flow tagging velocity measurements. Exp Fluids 20:142-152

Hoyas S, Jimenez J (2006) Scaling the velocity fluctuations in turbulent channels up to $\operatorname{Re}_{\tau}=2003$. Phys Fluids 18:011,702

$\mathrm{Hu}$ H, Koochesfahani M (2011) Thermal effects on the wake of a heater circular cylinder operating in mixed convection regime. J Fluid Mech 685:235-270

Hyland RW, Wexler A (1980) Formulations for the thermodynamic properties of the saturated phases of
$\mathrm{H}_{2} \mathrm{O}$ from $173.15 \mathrm{~K}$ to $473.15 \mathrm{~K}$. Tech. Rep. RP 216, Part 1, ASHRAE

Johansson A, Alfredsson P (1982) Effects of imperfect spatial resolution on measurements of wall-bounded turbulent shear flows. J Fluid Mech 122:295-314

Klewicki J, Falco R (1990) On accurately measuring statistics associated with small-scale structure in turbulent boundary layers using hot wire probes. J Fluid Mech 219:119

Klewicki J, Hill R (1998) Spatial structure of negative $\partial \tilde{u} / \partial y$ in a low $\mathrm{R} \theta$ turbulent boundary layer. J Fluid Eng 120:772-777

Klewicki J, Fife P, Wei T, McMurtry P (2007) A physical model of the turbulent boundary layer consonant with mean momentum balance structure. Phil Trans R Soc A 365:823-839

Koochesfahani M, Cohn R, Gendrich C, Nocera D (1997) Molecular Tagging Diagnostics for the study of kinematics and mixing in liquid phase flows. Chapter 2, Section 1. Developments in Laser Techniques and Fluid Mechanics., Springer-Verlag Berlin

Koochesfahani M, Cohn R, MacKinnon C (2000) Simultaneous whole-field measurements of velocity and concentration fields using combination of MTV and LIF. Meas Sci Tech 11:1289-1300

Koochesfahani MM, Nocera DG (2007) Handbook of Experimental Fluid Dynamics. Chapter 5.4: Molecular Tagging Velocimetry. Springer

Kwon Y, Philip J, de Silva CM, Hutchins N, Monty J (2014) The quiescent core of turbulent channel flow. J Fluid Mech 751:2228-254

Lee J, Kevin, Monty J, Hutchins N (2016) Validating under-resolved turbulence intensities for PIV experiments in canonical wall-bounded turbulence. Exp Fluids 57:129-140

Moffat RJ (1988) Describing the uncertainties in experimental results. Exp Thermal Fluid Sci 1:3-17

Monty J (2005) Developments in smooth wall turbulent duct flows. PhD thesis, The University of Melbourne, Australia

Monty J, Chong M (2009) Turbulent channel flow: comparison of streamwise velocity data from experiments and direct numerical simulation. J Fluid Mech 633:461-474

Moser RB, Kim J, Mansour NN (1999) Direct numerical simulation of turbulent channel flow up to $R e_{\tau}=590$. Phys Fluids 11(4):943-945

Nagashima A (1977) Viscosity of water substances New international formulation and its background. J Phys Chem Ref Data 6:1133-1166

Olson D, Katz A, Koochesfahani M, Rizzetta D, Visbal M (2013) On the challenges in experimental characterization of flow separation over airfoils at low 
Reynolds number. Exp Fluids 54:1470-1481

Olson D, Naguib A, Koochesfahani M (2015) Measurement of the wall pressure and shear stress distribution using molecular tagging velocimetry. Exp Fluids 56:171-177

Pouya S, Liu D, Koochesfahani M (2015) Effect of finite sampling time on estimation of Brownian fluctuation. J Fluid Mech 767:65-84

Ramsey M, Pitz R (2011) Template matching for improved accuracy in molecular tagging velocimetry. Exp Fluids 51:811-819

Sadr R, Klewicki JC (2003) An experimental investigation of the near-field flow development in coaxial jets. Phys Fluids 15(5):1233-1246

Savitzky A, Golay MJ (1964) Smoothing and differentiation of data by simplified least squares procedures. Analytical Chem 36(8):1627-1639

Smits A, Monty J, Hultmark M, Bailey S, Hutchins N, Marusic I (2011) Spatial resolution correction for wall-bounded turbulence measurements. J Fluid Mech 676:41-52

Sreenivasan KR (1989) Frontiers in Experimental Fluid Mechanics. Chapter: The turbulent boundary layer. Springer-Verlag

Thurlow EM, Klewicki JC (2000) Experimental study of turbulent Poiseuille-Couette flow. Phys Fluids 12(4):865-875

Wei T, Fife P, Klewicki JC, McMurtry P (2005) Properties of the mean momentum balance in turbulent boundary layer, pipe and channel flows. J Fluid Mech 522:303-327 


\section{University Library}

\section{- M M I N E R VA A gateway to Melbourne's research publications}

Minerva Access is the Institutional Repository of The University of Melbourne

Author/s:

Elsnab, JR;Monty, JP;White, CM;Koochesfahani, MM;Klewicki, JC

Title:

Efficacy of single-component MTV to measure turbulent wall-flow velocity derivative profiles at high resolution

Date:

2017-09-01

Citation:

Elsnab, J. R., Monty, J. P., White, C. M., Koochesfahani, M. M. \& Klewicki, J. C. (2017). Efficacy of single-component MTV to measure turbulent wall-flow velocity derivative profiles at high resolution. EXPERIMENTS IN FLUIDS, 58 (9), https://doi.org/10.1007/ s00348-017-2410-2.

Persistent Link:

http://hdl.handle.net/11343/283102 